$10 / 11^{3.99} y \leq D$

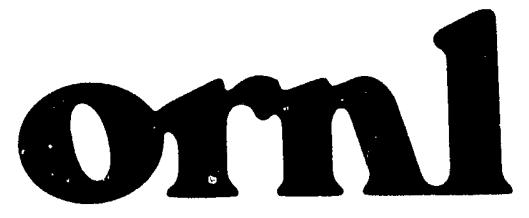

ORNL/TM-11903

OAK RIDGE

NATIONAL

LABORATORY

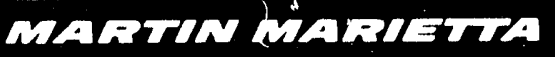

A Case Against a Divide and Conquer Approach to the Nonsymmetric Eigenvalue Problem

E. R. Jessup

MARTIN MARIETTA ENERGY SYSTEMS, INC. 
This report has been reproduced directly from the best available copy.

Available to DOE and DOE contractors from the Office of Scientific and Technical Information, P.O. Box 62, Oak Ridge, TN 37831; prices available from (615) 576-8401, FTS 626-8401.

Available to the public from the National Technical Information Service, U.S. Department of Commerce, 5285 Port Royal Rd., Springfield, VA 22161.

NTIS price codes-Printed Copy: AO7 Microfiche AO1

This report was prepared as an account of work sponsored by an agency of the United States Government. Neither the United States Government nor any agency thereof, nor any of their employees, makes any warranty, express or implied, or assumes any legal liability or responsibility for the accuracy, completeness, or usefulness of any information, apparatus, product, or process disclosed, or represents that its use would not infringe privately owned rights. Reference herein to any specific commercial product. process, or service by trade name, trademark, manufacturer, or otherwise, does not necessarily constitute or imply its endorsement, recommendation, or favoring by the United States Government or any agency thereof. The views and opinions of authors expressed herein do not necessarily state or reflect those of the United States Government or any agency theroof. 
Engineering Physics and Mathematics Division

Mathematical Sciences Section

\title{
A CASE AGAINST A DIVIDE AND CONQUER APPROACH TO THE NONSYMMETRIC EIGENVALUE PROBLEM
}

\author{
E.R. Jessup
}

Mathematical Sciences Section Oak Ridge National Laboratory

P.O. Box 2008, Bldg. 6012

Oak Ridge, TN 37831-6367

DATE PUBLISHED - DECEMBER 1991

Research was supported in part by the Applied Mathematical Sciences Research Program of the Office of Energy Research, U.S. Department of Energy and in part by National Science Foundation grant CCR-9109785.

Prepared by the Oak Ridge National Laboratory

Oak Ridge, T'ennessee 37831 managed by

Martin Marietta Energy Systems, Inc.

for the

U.S. DEPARTMENT OF ENERGY

under Contract No. DE-AC05-84OR21400 


\section{Contents}

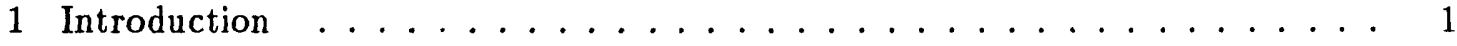

2 A Review of the Symmetric Tridiagonal Method . . . . . . . . . . . . 2

3 A Nonsymmetric Eigensolver . . . . . . . . . . . . . . . . 3

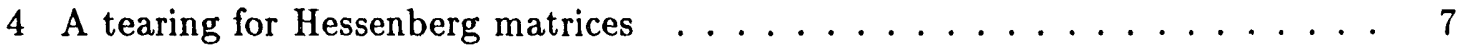

5 Obstacles to the Nonsymmetric Method . . . . . . . . . . . . . 9

5.1 Root-finding . . . . . . . . . . . . . . . . 9

5.2 Eigenvector Computation . . . . . . . . . . . . . 11

5.3 Deflation ...................... 12

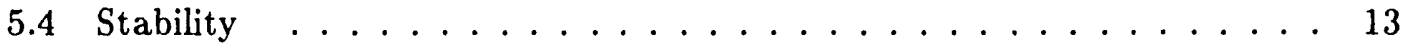

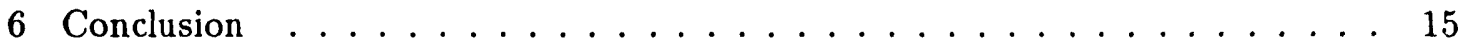

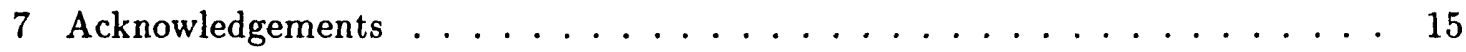

8 References ................................ 15 


\title{
A CASE AGAINST A DIVIDE AND CONQUER APPROACH TO THE NONSYMMETRIC EIGENVALUE PROBLEM
}

\author{
E.R. Jessup
}

\begin{abstract}
Divide and conquer techniques based on rank-one updating have proven fast, accurate, and efficient in parallel for the real symmetric tridiagonal and unitary eigenvalue problems and for the bidiagonal singular value problem. Although the divide and conquer mechanism can also be adapted to the real nonsymmetric eigenproblem in a straightforward way, most of the desirable characteristics of the other algorithms are lost. In this paper, we examine the problems of accuracy and efficiency that can stand in the way of a nonsymmetric divide and conquer eigensolver based on low-rank updating.
\end{abstract}




\section{Introduction}

The eigenvalues and eigenvectors of a real nonsymmetric matrix $A$ have traditionally been computed by first reducing $A$ to Hessenberg form $H$ and then computing the eigendecomposition of $H$ by the QR method. The serial nature of the QR method combined with the high cost of data transfer on distributed-memory multiprocessors has made parallel implementations of this approach inefficient [16]. The failure of parallel QR algorithms has sparked recent research into new algorithms including stabilized tridiagonalization $[9,14]$, iterative refinement techniques [11], and homotopy methods [25].

In this paper, we examine the use of divide and conquer techniques based on lowrank updating for solving the real nonsymmetric eigenvalue problem. This divide and conquer approach was first applied to the symmetric tridiagonal eigenvalue problem by Cuppen [8] and analyzed and implemented in parallel by Dongarra and Sorensen [12]. The algorithm involves tearing a symmetric tridiagonal matrix $T$ into a pair of symmetric tridiagonal submatrices $T_{1}$ and $T_{2}$ by removing off-diagonal elements of $T$ and using rank-one updating techniques to form the eigendecomposition of $T$ from those of $T_{1}$ and $T_{2}$. We review the method in Section 2 of this paper.

The symmetric divide and conquer technique of $[8,12]$ provides a fast and accurate serial alternative to the $Q R$ method or to bisection with inverse iteration $[12,23]$. In addition, the divide and conquer method is efficient when implemented on shared-memory multiprocessors [12]. Similar methods have also performed well for the bidiagonal singular value problem [24] and for the unitary eigenvalue problem [4]. In this paper, we show that the efficiency and accuracy shared by these divide and conquer methods cannot be expected in general when the approach is applied to the nonsymmetric eigenproblem.

In Sections 3 and 4 of this paper, we demonstrate how the symmetric divide and conquer method of $[8,12]$ can be extended in a straightforward way to nonsymmetric tridiagonal and Hessenberg eigenproblems if we assume that the submatrices formed from tearing are diagonalizable. This algorithm allows us to examine the troubles with a nonsymmetric divide and conquer eigensolver in direct analogy to the symmetric eigensolver. In [1], Adams and Arbenz consider a general rank-r update to a nonsymmetric matrix without assuming diagonalizability. Their proposed algorithm bears less resemblance to the symmetric algorithm, but many of the conclusions that we will draw about our simplified algorithm do apply to their complete theory. In fact, we will show that one major obstacle to accurate implementation of such a method arises for virtually any updating method that does not employ the original matrix $T$ at some stage after tearing. We discuss the difficulties that can plague a nonsymmetric divide and conquer method in Section 5. We present our conclusions in Section 6 .

Throughout this paper, unless otherwise specified, capital Greek and Roman letters represent matrices, lower case Roman letters represent column vectors, and lower case Greek letters represent scalars. A superscript $T$ denotes transpose, a superscript $H$ denotes conjugate transpose, and $\bar{h}$ is the element-wise complex conjugate of the vector $h$. The vector $e_{j}$ is the $j$-th "canonical vector" with all elements equal to zero except the $j$-th which equals 1 . 


\section{A Review of the Symmetric Tridiagonal Method}

An unreduced symmetric tridiagonal matrix $T$ of order $n=2 m$ can be written as the matrix sum

$$
T=\left(\begin{array}{cc}
T_{1} & \\
& T_{2}
\end{array}\right)+\theta \beta\left(\begin{array}{c}
e_{m} \\
\theta^{-1} e_{1}
\end{array}\right)\left(\begin{array}{cc}
e_{m}^{T} & \theta^{-1} e_{1}^{T}
\end{array}\right),
$$

where $\beta$ is the $m$ th off-diagonal element of $T, e_{i}$ is the $i$ th unit vector of length $m$, and $T_{1}$ and $T_{2}$ are symmetric tridiagonal of order $m$. The algorithm can be made backward stable with $\theta=\operatorname{sign}\left(e_{m}^{T} T_{1} e_{m}\right)$ [5].

If the solutions to the two smaller eigensystems are $T_{1}=X_{1} D_{1} X_{1}^{T}$ and $T_{2}=$ $X_{2} D_{2} X_{2}^{T}$, then

$$
T=X\left[D+\beta \theta\left(\begin{array}{c}
l_{1} \\
\theta^{-1} f_{2}
\end{array}\right)\left(\begin{array}{ll}
l_{1}^{T} & \left.\theta^{-1} f_{2}^{T}\right)
\end{array}\right) X^{T}\right.
$$

where

$$
X=\left(\begin{array}{ll}
X_{1} & \\
& X_{2}
\end{array}\right), \quad D=\left(\begin{array}{ll}
D_{1} & \\
& D_{2}
\end{array}\right),
$$

$l_{1}^{T}=e_{m}^{T} X_{1}$ is the last row of $X_{1}$, and $f_{2}^{T}=e_{1}^{T} X_{2}$ is the first row of $X_{2}$. To solve the eigenproblem for $T$, it is necessary to find the eigenvalues and eigenvectors of the diagonal plus rank-one matrix

$$
D+\rho z z^{T}=X^{T} T X
$$

where $z^{T}=\sqrt{\frac{\beta \theta}{\rho}}\left(l_{1}^{T} \theta^{-1} f_{2}^{T}\right)$, and $\rho$ is selected so that $\|z\|_{2}=1[8]$.

The eigensystem of $T$ is computed via the rank-one updating technique described in $[6,18]$. Namely, if all elements of $z$ are non-zero and if the diagonal elements of $D$ are distinct, then the eigenvalues of $D+\rho z z^{T}$ are the roots $\lambda_{1}>\ldots>\lambda_{n}$ of the secular equation

$$
w(\lambda)=1+\rho z^{T}(D-\lambda)^{-1} z=1+\sum_{j=1}^{n} \frac{\left(e_{j}^{T} z\right)^{2}}{\delta_{j}-\lambda} .
$$

If $\beta>0$ and the diagonal elements of $D$ are given by $\delta_{1}>\ldots>\delta_{n}$, each eigenvalue is bracketed by the adjacent diagonal elements of $D: \delta_{i}>\lambda_{i}>\delta_{i+1}$ and $\delta_{1}+\rho z^{T} z>\lambda_{1}>$ $\delta_{1}$. When $\beta<0$, a change of variables leads to a similar result. This interlacing property means that the roots of $w(\lambda)$ may be found efficiently using any one-dimensional root finder such as the one based on rational interpolation developed in [6]. Once $\lambda_{j}$ has been found, its corresponding eigenvector $u_{j}$ is computed from

$$
u_{j}=\frac{\left(D-\lambda_{j}\right)^{-1} z}{\left\|\left(D-\lambda_{j}\right)^{-1} z\right\|_{2}}
$$

When the diagonal elements of $D$ are not distinct, i.e., $\delta_{l}=\delta_{l+1}=\ldots=\delta_{l+k}$, the eigenproblem of order $n$ is reduced to one of order $n-k$ by the process of deflation. The eigenvector basis is first rotated to zero out the elements $\zeta_{l+1}, \ldots, \zeta_{l+k}$ corresponding 
to the multiple elements $\delta_{l+1}=\cdots=\delta_{l+k}$ : a product of plane rotations $G_{l}$ is applied so that

$$
G_{l}\left(\zeta_{l}, \zeta_{l+1}, \cdots, \zeta_{l+k}\right)^{T}=\left(\zeta_{l}^{\prime}, \zeta_{l+1}^{\prime}, \cdots, \zeta_{l+k}^{\prime}\right)^{T}=\left(\zeta_{l}^{\prime}, 0, \cdots, 0\right)^{T} .
$$

For $l+1 \leq j \leq l+k$, the $j$ th eigenvalue in exact arithmetic is the $j$ th element of $D\left(\lambda_{j}=\delta_{j}\right)$, and its corresponding eigenvector is the appropriate canonical vector $\left(u_{j}=e_{j}\right)[6]$.

Representing the product of all rotations by the matrix $G$, the matrix $T$ is expressed as $T=X G^{T} Q \Lambda Q^{T} G X^{T}=U \Lambda U^{T}$, where $U \Lambda U^{T}$ is the eigendecomposition of $G(D+$ $\left.\rho z z^{T}\right) G^{T}$. The eigenvalues of $T$ are the diagonal elements of $\Lambda$, and the eigenvectors of $T$ are the columns of $U=X G^{T} Q$.

The above derivation assumes exact arithmetic. Deflation rules have also been developed for finite precision in [12]: rotations are applied when diagonal elements of $D$ are close, and deflation occurs when elements of $z$ are small. Numerical experiments have confirmed that the increase in speed due to this denation is substantial for serial and shared-memory parallel implementations [12]. With appropriate choice of deflation criteria and use of extended precision, it is possible to guarantee computation of highly accurate eigenvalues and orthogonal eigenvectors [30].

Implementations of the divide and conquer method $[12,22]$ recursively subdivide the symmetric tridiagonal matrix $T$ until the resulting subproblems are of a desired order. In parallel implementations, the smallest subproblems are solved in parallel with one problem per processor, and the work to solve larger order subproblems is shared by more than one processor $[12,22]$. In particular, high parallel efficiency has been achieved on shared-memory multiprocessors by dynamically assigning independent root-finding and eigenvector computing tasks to separate processors at each level of updating [12]. A parallel implementation of the divide and conquer method can also be pipelined with reduction of a symmetric matrix to tridiagonal form $[10,12]$.

\section{A Nonsymmetric Eigensolver}

In this section, we investigate the application of low-rank updating techniques to the nonsymmetric tridiagonal eigenproblem. This problem arises as a subproblem in other numerical methods such as exponential interpolation $[2,3]$. Tridiagonal eigenproblems also result from nonsymmetric Lanczos algorithms [19] or the stabilized reduction of general eigenproblems to tridiagonal form $[9,15]$. We show in Section 4 that the theory we develop in this section for the tridiagonal problem extends in a straightforward manner to Hessenberg matrices. We assume the use of exact arithmetic in both cases. The nonsymmetric algorithms presented in this paper require that the submatrices be formed by the matrix tearing be diagonalizable.

The method of Section 2 was based on rank-one updating of a torn symmetric tridiagonal matrix. The theory behind that method does not carry through to solving a nonsymmetric updating problem $D+u v^{T}$. Furthermore, the updating matrix $u v^{T}$ is dense and otherwise unstructured. In this paper, we employ a rank-two tearing of a nonsymmetric matrix that leads to smaller order subproblems, a shorter recursion tree, and a convenient arrowhead updating matrix. A symmetric tridiagonal eigensolver 
based on rank-two updating appears to be competitive with the symmetric eirensolver based on rank-one updating [13]. Solution of symmetric arrowhead eigenproblems is discussed in $[28,31]$.

Let $T$ be the tridiagonal matrix with diagonal elements $\alpha_{1}, \ldots, \alpha_{n}$, suh-diagonal elements $\gamma_{1}, \ldots, \gamma_{n-1}$, and super-diagonal elements $\beta_{1}, \ldots, \beta_{n-1}$, and suppose $n=2 m+1$. By splitting off two superdiagonal elements $\beta_{m}$ and $\beta_{m+1}$ and the corresponding subdiagonal elements $\gamma_{m}$ and $\gamma_{m+1}$, we can write the matrix $T$ in terms of the tridiagonal submatrices $T_{1}$ and $T_{2}$ :

$$
T=\left(\begin{array}{l|l|l}
T_{1} & & \\
\hline & \alpha_{m} & \\
\hline & & T_{2}
\end{array}\right)+\left(\begin{array}{c|c|c} 
& \beta_{m} & \\
\hline \gamma_{m} & & \beta_{m+1} \\
\hline & \gamma_{m+1} &
\end{array}\right) .
$$

If $T_{1}$ and $T_{2}$ are diagonalizable, we can compute the eigendecompositions $T_{1}=X_{1} D_{1} X_{1}^{-1}$ and $T_{2}=X_{2} D_{2} X_{2}^{-1}$ with diagonal matrices $D_{1}$ and $D_{2}$. Substituting these decompositions and abbreviating $\alpha=\alpha_{m}$ gives the matrix product

$$
\begin{aligned}
T & =\left(\begin{array}{l|l|l}
X_{1} D_{1} X_{1}^{-1} & & \\
\hline & \alpha & \\
\hline & & X_{2} D_{2} X_{2}^{-1}
\end{array}\right)+\left(\begin{array}{l|l|l|l} 
& \beta_{m} & \\
\hline \gamma_{m} & & \beta_{m+1} \\
\hline & \gamma_{m+1} &
\end{array}\right) \\
& =\hat{X}\left[\begin{array}{l|l|l|l}
D_{1} & & \\
\hline & \alpha & \\
\hline & & D_{2}
\end{array}\right)+\left(\begin{array}{l|l|l} 
& v_{1} & \\
\hline h_{1}^{T} & & h_{2}^{T} \\
\hline & v_{2} &
\end{array}\right) \hat{X}^{-1},
\end{aligned}
$$

where

$$
\hat{X}=\left(\begin{array}{l|l|l}
X_{1} & & \\
\hline & 1 & \\
\hline & & X_{2}
\end{array}\right),
$$

$v_{1}=\beta_{m} X_{1}^{-1} e_{m}, v_{2}=\gamma_{m+1} X_{2}^{-1} e_{1}, h_{1}=\gamma_{m} X_{1}^{T} e_{m}$, and $h_{2}=\beta_{m+1} X_{2}^{T} e_{1}$ for canonical vectors $e_{1}$ and $e_{m}$ of appropriate length.

We permute the elements of equation (2) to form

$$
T=X\left[\left(\begin{array}{cc|c}
D_{1} & & \\
& D_{2} & \\
\hline & & \alpha
\end{array}\right)+\left(\begin{array}{ll|l} 
& & v_{1} \\
& v_{2} \\
\hline h_{1}^{T} & h_{2}^{T} & 0
\end{array}\right)\right] X^{-1}
$$

with

$$
X=\left(\begin{array}{ll|l}
X_{1} & & \\
& X_{2} & \\
\hline & & 1
\end{array}\right)
$$

and rewrite the interior matrix of equation (3) as 


$$
M=\left(\begin{array}{cc|c}
D_{1} & & \\
& D_{2} & \\
\hline & \alpha
\end{array}\right)+\left(\begin{array}{cc|c} 
& & v_{1} \\
& & v_{2} \\
\hline h_{1}^{T} & h_{2}^{T} & 0
\end{array}\right)=\left(\begin{array}{cc}
D & v \\
h^{T} & \alpha
\end{array}\right) .
$$

The arrowhead matrix $M$ is the sum of a diagonal matrix and a rank two nonsymmetric matrix. The eigenvaiues of the matrix $M$ are the eigenvalues of $T$. The left and right eigenvectors of $M$ premultiplied by $X^{-H}$ and $X$, respectively, are the left and right eigenvectors of $T$.

The procedure for computing the remaining eigenvalues and eigenvectors of $M$ follows basic steps similar to those for the eigendecomposition of a diagonal plus symmetric rank one matrix developed in $[6,18]$, but because $M$ is nonsymmetric, the details differ in several important ways.

If $M$ has diagonal elements $\delta_{1}, \ldots, \delta_{n-1}, \alpha$, last column $\left(v_{1}, \ldots, v_{n-1}, \alpha\right)^{T}$, and last row $\left(h_{1}, \ldots, h_{n-1}, \alpha\right)$, then the following lemmas establish when diagonal elements of $M$ can be retained as eigenvalues of $T$.

Lemma 3.1. If the diagonal elements $\delta_{1}, \ldots, \delta_{n-1}$ of $M$ are distinct, the element $\delta_{j}$ is an eigenvalue of $M$ if and only if $v_{j} h_{j}=0$.

Lemma 3.2. If $M$ has repeated diagonal elements $\delta_{i}=\delta_{j}$ with $h_{i} h_{j} \neq 0, M$ is similar to a matrix with $\delta_{i}=\delta_{j}$ and $h_{i}=0$.

Proof: The proof is by construction of the unitary similarity transformation that reduces $h_{i}$ to zero when $\delta_{i}=\delta_{j}$. Let

$$
\tau^{2}=\left|h_{j}\right|^{2}+\left|h_{i}\right|^{2}, \quad c=\frac{h_{j}}{\tau}, \quad s=\frac{h_{i}}{\tau},
$$

then the matrix is transformed in the following way

$$
\left(\begin{array}{ccc}
\bar{c} & -\bar{s} & 0 \\
s & c & 0 \\
0 & 0 & 1
\end{array}\right)\left(\begin{array}{ccc}
\delta_{i} & 0 & v_{i} \\
0 & \delta_{j} & v_{j} \\
h_{i} & h_{j} & \alpha
\end{array}\right)\left(\begin{array}{ccc}
c & \bar{s} & 0 \\
-s & \bar{c} & 0 \\
0 & 0 & 1
\end{array}\right)=\left(\begin{array}{ccc}
\delta_{i} & 0 & \bar{c} v_{i}-\bar{s} v_{j} \\
0 & \delta_{j} & s v_{i}+c v_{j} \\
0 & \tau & \alpha
\end{array}\right)
$$

Similarly, if $\delta_{i}=\delta_{j}$ and $v_{i} v_{j} \neq 0, M$ is similar to a matrix with $\delta_{i}=\delta_{j}$ and $v_{i}=0$. If $h_{i}=0, e_{i}$ is the right eigenvector of $M$ corresponding to the eigenvalue $\delta_{i}$. If $v_{i}=0, e_{i}$ is the left eigenvector of $M$ corresponding to the eigenvalue $\delta_{i}$.

We first consider the case where $D$ has distinct diagonal elements $\delta_{1} \neq \delta_{2} \ldots \neq \delta_{n}$ and $v$ and $h$ have no zero elements. By Lemmas 3.1 and 3.2, no diagonal element $\delta_{1}, \ldots, \delta_{n-1}$ can be an eigenvalue of $M$. The eigenvalues of $M$ are then the roots of the complex rational equation

$$
g(\lambda)=(\lambda-\alpha)+\sum_{i=1}^{n-1} \frac{\left(e_{i}^{T} h\right)\left(e_{i}^{T} v\right)}{\delta_{i}-\lambda}=0
$$


The right eigenvector $q$ of $M$ associated with eigenvalue $\lambda$ is given by

$$
q=\left(\begin{array}{c}
\hat{q} \\
\xi
\end{array}\right)=\left(\begin{array}{c}
-(D-\lambda I)^{-1} v \\
1
\end{array}\right) \xi
$$

and the left eigenvec ${ }^{4}$ or is

$$
s=\left(\begin{array}{l}
\hat{s} \\
\zeta
\end{array}\right)=\left(\begin{array}{c}
-(D-\lambda)^{-H} \bar{h} \\
1
\end{array}\right) \zeta
$$

where $\xi, \zeta$ are chosen to make $s_{j}^{H} q_{j}=1$. (This 1 crmalization ensures that the left eigenvector matrix is the inverse of the right eigenvecior matrix.)

When there are zero elements in $v$ or $h$, the riatrix $(D-\lambda I)$ is singular, and equations (5) and (6) cannot be used to compute the cygnvectors of $M$. To derive rules for computing eigenvectors in this case, we first suppr e that $M$ has been transformed by a series of elementary transformations accumulated into the matrix $G$ so that equal diagonal elements correspond to zero elements of $v$ or $h$. We then permute the $n \times n$ matrix $M \leftarrow P G M G^{*} P^{T}$ so that all zero elements in its last row or column are grouped together as follows:

$$
M=\left(\begin{array}{cccc}
\hat{D}_{0} & 0 & 0 & 0 \\
0 & \hat{D}_{1} & 0 & V \\
0 & 0 & \hat{D}_{2} & 0 \\
0 & 0 & H & \hat{M}
\end{array}\right)
$$

with

$$
H=\left(\begin{array}{c}
0 \\
\tilde{h}^{T}
\end{array}\right) \in \mathcal{C}^{r \times p}, \quad V=\left(\begin{array}{cc}
0 & \tilde{v}
\end{array}\right) \in \mathcal{C}^{t \times r}
$$

where $\tilde{v}$ and $\tilde{h}$ have no zero elements. We further assume that the similarity transformations have been applied so that $\hat{D}_{1}$ and $\hat{D}_{2}$ have no common diagonal elements. Thus, no eigenvalue of $\hat{D}_{1}$ is an eigenvalue of $\hat{D}_{2}$ or of $\hat{M}$.

The zero structure of $M$ allows us to deflate out some eigenpairs. Specifically, The diagonal elements of the diagonal submatrix $\hat{D}_{0} \in \mathcal{C}^{j \times j}$ are eigenvalues of the matrix $M$ with corresponding left and right eigenvectors $\left(I_{j}, 0,0,0\right)^{T}$. The diagonal elements of $\hat{D}_{1} \in \mathcal{C}^{t \times t}$ are eigenvalues of $M$ with right eigenvectors $\left(\begin{array}{llll}0 & I_{t} & 0 & 0\end{array}\right)^{T}$. The eigenvalues of $\hat{D}_{2} \in \mathcal{C}^{p \times p}$ are eigenvalues of $M$ with corresponding left eigenvectors $\left(0,0, \quad I_{p}, 0\right)^{T}$. The remaining eigenvalues of $M$ are the eigenvalues of $\hat{M}$. By Lemmas 3.1 and 3.2 , no eigenvalue of $\hat{D}_{1}$ or $\hat{D}_{2}$ can also be an eigenvalue of $\hat{M}$.

To compute the remaining eigenvectors, we first determine the eigenvectors of the submatrix

$$
M_{H}=\left(\begin{array}{cc}
\hat{D}_{2} & 0 \\
H & \hat{M}
\end{array}\right)
$$


The right eigenvector $\left(\hat{q}_{1}^{T}, \hat{q}_{2}^{T}\right)^{T}$ corresponding to the eigenvalue $\lambda$ satisfies

$$
\begin{aligned}
\left(\hat{D}_{2}-\lambda\right) \hat{q}_{1} & =0 \\
H \hat{q}_{1}+(\hat{M}-\lambda) \hat{q}_{2} & =0 .
\end{aligned}
$$

If $\lambda$ is not a diagonal element of $\hat{D}_{2}$, then $\hat{q}_{1}=0,(\hat{M}-\lambda) \hat{q}_{2}=0$, and $\hat{q}_{2}$ is the right eigenvector of $\hat{M}$ determined from equation (5). The corresponding left eigenvector $\left(\hat{s}_{1}^{T}, \hat{s}_{2}^{T}\right)^{T}$ satisfies

$$
\begin{aligned}
\hat{s}_{2}^{H}(\hat{M}-\lambda) & =0 \\
\hat{s}_{1}^{H}\left(\hat{D}_{1}-\lambda\right)+\hat{s}_{2}^{H} H & =0 .
\end{aligned}
$$

The vector $\hat{s}_{2}$ is found using equation (6), and the vector $\hat{s}_{1}$ comes from a diagonal scaling of $s_{2}^{H} H$. If $\lambda$ is the $k$ th diagonal element of $\hat{D}_{2}$, then $\hat{q}_{1}=e_{k},(\hat{M}-\lambda) \hat{q}_{2}=$ $-H e_{k}$, and $\hat{q}_{2}$ can be found by solving the latter linear system. The corresponding left eigenvector is the appropriate canonical vector. Note that for the arrowhead matrix $\hat{M} \in \mathcal{C}^{r \times r}$, any left or right eigenvector of $M_{H}$ can be found $O(r)$ operations.

We then determine the eigenvectors of the matrix

$$
M_{V}=\left(\begin{array}{ccc}
\hat{D}_{1} & 0 & V \\
0 & \hat{D}_{2} & 0 \\
0 & H & \hat{M}
\end{array}\right)
$$

from those of $M_{H}$. The left eigenvector $\left(s_{1}^{T}, s_{2}^{T}\right)^{T}$ of $M_{V}$ corresponding to the eigenvalue $\lambda$ satisfies

$$
\begin{aligned}
s_{1}^{H}\left(\hat{D}_{1}-\lambda\right) & =0 \\
s_{1}^{H}(0, V)+s_{2}^{H}\left(M_{H}-\lambda\right) & =0
\end{aligned}
$$

If $\lambda$ is not a diagonal element of $\hat{D}_{1}, s_{1}^{H}=0$, and $s_{2}^{H}$ is just a left eigenvector of $M_{H}$. Thie corresponding right eigenvector $\left(q_{1}^{T}, q_{2}^{T}\right)^{T}$ of $M$ satisfies

$$
\begin{aligned}
\left(\hat{D}_{1}-\lambda\right) q_{1}+(0, V) q_{2} & =0 \\
\left(M_{H}-\lambda\right) q_{2} & =0
\end{aligned}
$$

and $q_{2}$ is the associated right eigenvector of $M_{H}$. The vector $q_{1}$ is produced by a diagonal scaliatg of $(0, V) q_{2}$. If $\lambda$ is the $k$ th diagonal element of $\hat{D}_{1}, s_{1}^{H}=e_{k}$, and $s_{2}^{H}$ is found by solving the arrowhead system $s_{2}^{H}\left(M_{H}-\lambda\right)=-e_{k}^{H}(0, V)$ in $O(r+t)$ operations. The corresponding right eigenvector is the appropriate canonical vector.

The eigenvectors of $M$ are those of $M_{V}$ and of $D_{3}$ with zeros appended or prepended as necessary.

\section{A tearing for Hessenberg matrices}

In this section, we show how the divide and conquer scheme of Section 3 can be applied to an upper Hessenberg matrix $H$ of order $n=2 m+1$, under the assumption that 
the Hessenberg submatrices formed by matrix tearing are diagonalizable. If $H_{1}=$ $X_{1} D_{1} X_{1}^{-1}$ and $H_{2}=X_{2} D_{2} X_{2}^{-1}$, then

$$
\begin{aligned}
& H=\left(\begin{array}{l|l|l}
H_{1} & & \mathrm{Z} \\
\hline & \alpha_{m} & \\
\hline & & H_{2}
\end{array}\right)+\left(\begin{array}{c|c|c} 
& v & \\
\hline \gamma_{m} & & h^{T} \\
\hline & \gamma_{m+1} &
\end{array}\right) \\
& =X\left[\left(\begin{array}{c|c|c}
D_{1} & & \\
\hline & \alpha & \\
\hline & & D_{2}
\end{array}\right)+\left(\begin{array}{c|c|c} 
& X_{1}^{-1} v & X_{1}^{-1} Z X_{2} \\
\hline \gamma_{m} e_{m}^{T} X_{1} & & h^{T} X_{1} \\
\hline & \gamma_{m+1} X_{2}^{-1} e_{1} &
\end{array}\right)\right] X^{-1}
\end{aligned}
$$

with $\alpha=\alpha_{m}$ and $X$ the direct sum $X_{1} \oplus 1 \oplus X_{2}$. The interior matrix can be permuted to form

$$
M=\left(\begin{array}{c|c|c}
D_{1} & X_{1}^{-1} Z X_{2} & v_{1} \\
\hline & D_{2} & v_{2} \\
\hline h_{1}^{T} & h_{2}^{T} & \alpha
\end{array}\right)
$$

where $v_{1}=X_{1}^{-1} v, v_{2}=\gamma_{m+1} X_{2}^{-1} e_{1}, h_{1}=\gamma_{m} X_{1}^{T} e_{m}$, and $h_{2}=X_{2}^{T} h$. The eigenvalues of $M$ not equal to diagonal elements of $D_{1}$ and $D_{2}$ are the roots of the secular equation

$$
\begin{aligned}
r(\lambda)= & (\alpha-\lambda)+h_{1}^{T}\left(D_{1}-\lambda I\right)^{-1} v_{1}+h_{2}^{T}\left(D_{2}-\lambda I\right)^{-2} v_{2}- \\
& h_{1}^{T}\left(D_{1}-\lambda I\right)^{-1}\left(X_{1}^{-1} Z X_{2}\right)\left(D_{2}-\lambda I\right)^{-1} v_{2} .
\end{aligned}
$$

Note that $r(\lambda)=0$ has the same form as the tridiagonal secular equation (4) plus an additional cross term involving $Z$.

The right eigenvector of $M$ for eigenvalue $\lambda$ is

$$
q=\left(\begin{array}{c}
q_{1} \\
q_{2} \\
\zeta
\end{array}\right)=\zeta\left(\begin{array}{c}
-\left(D_{1}-\lambda I\right)^{-1}\left[-\left(X_{1}^{-1} Z X_{2}\right)\left(D_{2}-\lambda I\right)^{-1} v_{2}+v_{1}\right] \\
-\left(D_{2}-\lambda I\right)^{-1} v_{2} \\
1
\end{array}\right)
$$

The deflation rules for the right eigenvectors of $M$ derive from this expression (using $M q=\lambda q$ ) and are not as simple as those given for the tridiagonal case in Lemmas 3.1 and 3.2. For example, a diagonal element of $D_{1}$ is retained as an eigenvalue $\lambda$ of $M$ whenever $D_{2}-\lambda I$ is nonsingular, and both of the following relations are satisfied:

$$
\begin{aligned}
\zeta e_{j}^{T} v_{1} & =\zeta e_{j}^{T}\left(X_{1}^{-1} Z X_{2}\right)\left(D_{2}-\lambda^{-1} v_{2}\right. \\
h_{1}^{T} q_{1} & =\zeta\left[h_{2}^{T}\left(D_{2}-\lambda I\right)^{-1} v_{2}+(\alpha-\lambda)\right]
\end{aligned}
$$

for $e_{j}^{T}\left(D_{1}-\lambda I\right)=0$ and any choice of $q_{2}$. The requirements are similarly complicated if $\lambda$ is a diagonal element of $D_{2}$ but not of $D_{1}$. If $\lambda$ appears in both $D_{1}$ ( $j$ th diagonal element) and $D_{2}$ ( $k$ th diagonal element), the requirements simplify to

$$
\begin{aligned}
-\zeta e_{j}^{T} v_{1} & =e_{j}^{T}\left(X_{1}^{-1} Z X_{2}\right) q_{2} \\
\zeta e_{k}^{T} v_{2} & =0
\end{aligned}
$$


for any $q_{1}$. The formulas for the ieft eigenvector and deflation rules resemble those for the right eigenvector. The divide and conquer mechanism thus extends to the Hessenberg case, although less simply and less efficiently than to the tridiagonal case.

\section{Obstacles to the Nonsymmetric Method}

To this poinr, we have shown that as long as the submatrices formed from tearing are diagonalizable, a divide and conquer method for nonsymmetric tridiagonal and Hessenberg eigenproblems can be derived aloi $I$ the lines of the symmetric method reviewed in Section 2. In the remainder of this paper, we discuss difficulties with efficiency and stability that stand in the way of a practical implementation of a nonsymmetric divide and conquer method. We use our method and that of [1] to illustrate the problems, but many of our deliberations would apply to any divide and conquer algorithm of this type.

\subsection{Root-finding}

An accurate and efficient root-finder is essential to the success of the symmetric divide and conquer method of $[8,12]$. The nonsymmetric tridiagonal secular equation (4) $g(\lambda)=0$, however, shares few of the properties that make the symmetric secular equation $(2) w(\lambda)=0$ so easy to sclve. The nonsymmetric function $g(\lambda)$ can still be evaluated in $O(n)$ flops, but because it derives from the spectral decompositions of $T_{1}$ and $T_{2}$, it can have complex coefficients. The complex roots of $g(\lambda)=0$ occur in conjugate pairs but otherwise can lie anywhere within the union of Gerschgorin disks of $T$ or of $M$. Only the poles $\delta_{1}, \ldots, \delta_{n-1}$ of $g(\lambda)$ are easily identified. Unlike the symmetric equation, where the roots interlace the poles, there is no obvious connection between the locations of the poles and roots of $g(\lambda)=0$. The only advantage of the nonsymmetric equation is that only its roots with nonnegative (or nonpositive) imaginary roots need be explicitly computed to determine the full spectrum.

To illustrate the general structure of $g(\lambda)$ we present contour plots of $\log (|g(\lambda)|)$ over the area of the complex plane containing all roots and poles for two $5 \times 5$ matrices. Figure 1 shows the function for matrix $T_{5}$ having diagonal elements $1,2,3,4,5$ and off-diagonal elements $\beta_{k}=1, \gamma_{k}=-1, k=1, \ldots, 4$. Boxes mark the four complex poles of the function, and $\times$ 's mark its one real and four complex roots. In this case, the structure of $|g(\lambda)|$ is quite regular. Figure 2 shows $\log (|g(\lambda)|)$ for a $5 \times 5$ random tridiagonal matrix. In this instance, two poles and three roots lie on the real line. The remaining complex conjugate poles and roots are nearly coincident, although deflation ensures that they are not equal. (In both cases, the function rises monotonically toward the poles: apparent structure near the poles is an artifact of the printer's resolution.)

In this section, we examine the possibility of unconditional global convergence to all roots of $g(\lambda)=0$. As the roots and poles of this equation do not interlace, the first order of business is to localize the roots. To this end, we present Weyl's algorithm [20] which was originally devised for polynomials. Given an initial search interval for the roots (here, the union of all Gerschgorin disks of $T$ or of the deflated matrix $M$ ), we can 
cover it with closed squares and determine the number of roots lying within each. Any squares found to contain no roots are discarded from the search area. The remaining squares are subdivided and the process is repeated recursively with the smaller squares. (Lehmer's method uses a similar process that covers the search area with disks [20, 21]. Derwidués method covers the upper half plane with strips [21].)

To implement Weyl's algorithm, it remains to devise an exclusion test to determine which squares are empty [20]. Let $\Gamma$ be the boundary of a square to be tested. Because we know the locations of the poles of $g(\lambda)$, we could determine the number of roots inside of $\Gamma$ by numerically evaluating the winding number

$$
n=\frac{1}{2 \pi i} \int_{\Gamma} \frac{g^{\prime}(\lambda)}{g(\lambda)} d \lambda
$$

which equals the number of zeros of $g(\lambda)$ inside $\Gamma$ minus the number of poles of $g(\lambda)$ inside $\Gamma$ [7]. The cost of computing this integral can be high, however, and it may be impossible to attain an accurate result when zeros lie close to $\Gamma$ [20]. An alternative is to see whether $\max _{\Gamma}|g(\lambda)|$ ever exceeds $\max _{\Gamma}\left|g^{\prime}(\lambda)\right| \sqrt{\omega}$ on a square $\Gamma$ of side $\omega$ not enclosing a pole. If it does, the function cannot have a zero inside of $\Gamma$ [7]. (A similar test is described in [20] for polynomials.) Determining these maxima amounts to yet another root-finding or optimization problem and so would be expensive. Such a test could also fail for roots near poles or near the edge of a square.

Even if a practical exclusion test could be devised, Weyl's method offers only linear convergence. Furthermore, Weyl's algorithm requires that significant work be performed on intervals containing no eigenvalues. (In the symmetric case, the interlacing property means that only intervals of the real line containing eigenvalues are examined $[12,22]$.) Weyl's algorithm does lend itself to a parallel implementation as each square can be examined independently by one processor, although experiments with one-dimensional multisection routines suggest that an efficient mechanism for dynamically assigning squares to processors would be required for good performance $[22,26]$. According to Henrici [20], this sort of multisection procedure is the only way to compute all roots of a polynomial to a desired precision with unconditional global convergence. We are not aware of any other globally convergent method for computing all zeros of rational functions.

While it appears that there is no reasonably efficient globally convergent method for solving the secular equation, alternatives such as Newton's method, Bernoulli's method, Graeffe's root squaring method, or Rutishauser's quotient-difference method $[20,21]$ might work in practice. We could also pose the root-finding problem as the problem of finding all local minimizers of $|g(\lambda)|$ within the minimal set of Gerschgorin disks of $T$ or $M$. As a consequence of the Maximum Modulus Theorem [7], the modulus $|g(\lambda)|$ has no local minimizers other than its roots. In this case, we can use the method of steepest descent for guaranteed convergence to a single root $[17,20]$. Any of these methods would require a mechanism for choosing starting guesses for each root and a mechanism for determining if all roots have been computed.

Finally, we note that the $O(n)$ cost for evaluating $g(\lambda)$ results from having a tridiagonal matrix torn into diagonalizable submatrices. In contrast, when evaluating the 
Hessenberg secular equation (9), we must first determine $X_{1}^{-1} Z X_{1}$ in $O\left(m^{3}\right)$ operations then evaluate $r(\lambda)=0$ in $O\left(m^{2}\right)$ operations where $m=\frac{n-1}{2}$. If the submatrices resulting from tearing a tridiagonal or Hessenberg matrix, are not diagonalizable, the cost of evaluating the resulting secular equation is also higher than for $g(\lambda)$ or $r(\lambda)$, respectively [1].

\subsection{Eigenvector Computation}

The derivation in Section 3 shows that, for an $n \times n$ matrix $M$, all left and right eigenvectors are produced by our nonsymmetric divide and conquer algorithm in $O\left(n^{2}\right)$ operations. Furthermore, at each stage, every eigenvector of $M_{H}, M_{V}$, or $M$ is computed independently of every other. Although independent computation of eigenvectors is good for parallel efficiency, it may not be the best numerical strategy for the nonsymmetric eigenproblem. To compute an accurate singular value decomposition of a matrix by the divide and conquer strategy of [24], it is necessary to compute each right singular vector from its corresponding left singular vector rather than by independent formulas. In part, this ensures correct pairing of left and right singular vectors corresponding to close singular values. Similarities between the divide and conquer algorithms for the SVD and the nonsymmetric eigenproblem suggest than an accurate eigensolver for the latter might also require the right eigenvectors to he computed from the left eigenvectors.

In the SVD algorithm of [24], the right singular vector is given by a diagonal scaling of the left singular vector. The relationship between the left and right singular vectors that makes this efficient transformation possible does not carry through to the nonsymmetric eigenproblem. If the matrix $\hat{M}$ in equation (7) is diagonalizable, the matrix of right eigenvectors $Q$ can be computed from the left eigenvectors only by inverting the latter in $O\left(r^{3}\right)$ operations. If the eigendecomposition $M_{H}=Q_{H} \Lambda_{H} Q_{H}^{-1}$ is computed stably, the left eigenvectors of the diagonalizable matrix $M_{V}$ derive from

$$
M_{V}=\left(\begin{array}{cc}
\hat{D}_{1} & V \\
0 & M_{H}
\end{array}\right)
$$

and the relation

$$
\Lambda=\left(\begin{array}{cc}
\hat{D}_{1} & V \\
0 & \Lambda_{H}
\end{array}\right)=\left(\begin{array}{cc}
I & A \\
0 & Q_{H}^{-1}
\end{array}\right)\left(\begin{array}{cc}
\hat{D}_{1} & 0 \\
0 & M_{H}
\end{array}\right)\left(\begin{array}{cc}
I & C \\
0 & Q_{H}
\end{array}\right)
$$

In this case, $A=-C Q_{H}^{-1}$, and each element of $C$ is computed by

$$
e_{j}^{T} C e_{k}=-\left(e_{j}^{T} V\right)\left(Q_{H}^{-1} e_{k}\right) /\left(e_{j}^{T} \hat{D}_{1} e_{j}-e_{k}^{T} \Lambda_{H} e_{k}\right),
$$

$1 \leq j \leq p$ and $1 \leq k \leq r+t$. Computing the submatrix $C$ takes $O(p(r+t))$ operations, but computing $A$ takes $O\left(p(r+t)^{2}\right)$ operations. The matrix product needed to compute $A$ and the inversion of $Q^{-1}$ make the eigenvector computation expensive both serially and in parallel. In the algorithm of [1], the left and right eigenspaces are computed independently. 


\subsection{Deflation}

In practice, the cost of the symmetric divide and conquer method of $[8,12]$ is strongly problem dependent and is determined by the amount of deflation occurring for a given problem $[12,23]$. When deflation is prevalent, divide and conquer is the fastest way to compute accurately all eigenvalues and eigenvectors of a symmetric tridiagonal matrix, but if no deflation occurs, the divide and conquer and QR methods are of comparable cost [23]. We have not done experiments to determine the likelihood of deflation in the nonsymmetric case but do note that the zero structure of the computed eigenvector matrices could influence the savings. For symmetric matrices, the eigenvector matrix decouples into the direct sum of an identity matrix and a computed submatrix, but for nonsymmetric matrices, the eigenvector matrices have nonsymmetric zero structure and, hence, possibly fewer zeros than if $v=h$. Fewer zeros would mean less savings when the eigenvectors of $M$ are computed and and when they are backtransformed to those of the original matrix $T$.

As in the symmetric case, deflation rules can be formulated for use in a finite precision implementation. A diagonal element $\delta_{j}$ of the matrix $M$ is retained as an eigenvalue of $M$ when $\delta_{j}$ is close to some other diagonal element $\delta_{i}$ or when an element $v_{i}$ or $h_{i}$ is small. In the first instance, similarity transformations are applied as in Lemma 3.2 to zero out a border element $h_{j}$ or $v_{j}$ corresponding to one duplicate diagonal element. Error analysis of this process proceeds much as for the symmetric case in $[6,12]$ and shows that finite precision deflation leads us to work with a matrix $M+E$ close to the one we would use in exact arithmetic.

If we accept the eigendecomposition of $M+E$ as the eigendecomposition of $M$, we can then see an error in a computed eigenvalue $\lambda_{i}$ of $M$ as large as [19, 31]

$$
\left|\lambda_{i}(M)-\lambda_{i}(M+E)\right| \approx \frac{|E|}{\sigma\left(\lambda_{i}(M)\right)},
$$

where $1 / \sigma\left(\lambda_{i}\right)$ is the condition number of the eigenvalue $\lambda_{i}$. Thus, if all eigenvalues of $M$ are well conditioned, deflation of this sort should pose no threat to the accuracy of the method. Similarly, a well-conditioned eigenvector would not be sensitive to the small errors introduced by the similarity transformations.

Ignoring small elements of $h$ and $v$ also appears to have little influence on the accuracy of the solution as measured by the residual error. We accept $\left(\delta_{i}, e_{i}\right)$ as a right eigenpair of $M$ whenever $\left|h_{i}\right| \leq$ tol $=\theta \epsilon_{M}\|M\|$ with $\theta=O(1)$ and $\epsilon_{M}=$ machine epsilon. This ensures that the residual error of the eigenpair is bounded above by a small value

$$
\left\|M e_{i}-\delta_{i} e_{i}\right\|=\left|\left(h^{T} e_{i}\right) e_{n}\right|=\left|h_{i}\right| \leq \text { tol }
$$

These resuits indicate that deflation should not influence the accuracy of well-conditioned eigenpairs but could be problematic for ill-conditioned eigenpairs.

The time savings due to deflation are difficult to predict. The nonsymmetric zero structure of the eigenvector matrices leads to reduced savings in both computation and backtransformation of the eigenvectors of $M$ when compared to deflation in the symmetric method. The loss of the efficient root-finder, however, suggests that eigenvalue 
computation time may occupy a substantially larger fraction of the total time than it does in the symmetric case. If this is true, the savings in eigenvalue computation following deflation would be more important for nonsymmetric than for symmetric matrices.

The algorithm of [1] attains a low operation count by computing the eigenvectors of $T$ through direct system solution rather than by computing and backtransforming the eigenvectors of an intermediate matrix. Adams and Arbenz conclude that as long as the required system solution is stable, deflation of the problem is unnecessary. (The stability of the computation is as yet unestablished.)

\subsection{Stability}

There remains one additional aspect of a divide and conquer method that can most severely impact its accuracy even if it allows for defective submatrices. Namely, if either of the torn submatrices $T_{1}$ or $T_{2}$ of a nonsymmetric matrix $T$ is ill-conditioned with respect to the eigenproblem, the accuracy of its computed eigendecomposition can be poor $[19,31]$. Because the eigendecomposition of $T$ is built up from those of $T_{1}$ and $T_{2}$ and is never corrected using $T$ itself, the eigendecomposition of $T$ will also be inaccurate.

The following example demonstrates that even a matrix that is well-conditioned for eigendecomposition can have ill-conditioned submatrices. The Hessenberg matrix

$$
H(\delta)=\left(\begin{array}{ccccccccc}
0 & 0 & 0 & 10^{-8}+\delta & 0 & 0 & 0 & 0 & 1 \\
1 & 0 & 0 & 0 & 0 & 0 & 0 & 0 & 1 \\
0 & 1 & 0 & 0 & 0 & 0 & 0 & 0 & 1 \\
0 & 0 & 1 & 0 & 0 & 0 & 0 & 0 & 1 \\
0 & 0 & 0 & 1 & 0 & 0 & 0 & 0 & 1 \\
0 & 0 & 0 & 0 & 1 & 0 & 0 & 0 & 1 \\
0 & 0 & 0 & 0 & 0 & 1 & 0 & 0 & 1 \\
0 & 0 & 0 & 0 & 0 & 0 & 1 & 0 & 1 \\
0 & 0 & 0 & 0 & 0 & 0 & 0 & 1 & 1
\end{array}\right)
$$

has distinct eigenvalues all with condition numbers near 10 and an eigenvector matrix with 2 -norm condition number of 3.4 when $\delta=0$. Like $H(0)$, the $4 \times 4$ submatrix formed by rank-two tearing

$$
H_{2}=\left(\begin{array}{llll}
0 & 0 & 0 & 1 \\
1 & 0 & 0 & 1 \\
0 & 1 & 0 & 1 \\
0 & 0 & 1 & 1
\end{array}\right)
$$

has distinct eigenvalues with condition numbers near 10 and an eigenvector matrix with 2-norm condition number 2.0. However, the submatrix

$$
H_{1}(\delta)=\left(\begin{array}{cccc}
0 & 0 & 0 & 10^{-8}+\delta \\
1 & 0 & 0 & 0 \\
0 & 1 & 0 & 0 \\
0 & 0 & 1 & 0
\end{array}\right)
$$


has four distinct eigenvalues with condition numbers around $10^{5}-10^{6}$ and an eigenvector condition number of about $10^{6}$ for $\delta$ near or equal to zero. (See [31].)

To demonstrate the sensitivity of $H(\delta)$ to the ill-condition of $H_{1}(\delta)=X_{1}(\delta) D_{1}(\delta) X_{1}(\delta)^{-1}$ in our divide and conquer algorithm, we first compute the structured matrix

$$
M(\delta)=\left(\begin{array}{c|c|c}
D_{1}(\delta) & X_{1}(\delta)^{-1} Z X_{2} & v_{1}(\delta) \\
\hline & D_{2} & v_{2} \\
\hline h_{1}(\delta)^{T} & h_{2}^{T} & \alpha
\end{array}\right)
$$

from equation (8) using Matlab [27] for $\delta=0$ and $\delta=\epsilon_{M}=2.22 \times 10^{-16}$. All eigendecompositions needed to construct and analyze $M$ are computed in double precision using the Matlab function eig which computes the eigenvalues by the QL method $[29,27]$.

The small perturbation in $H(\delta)$ leads to a difference in $M(\delta)$ of

$$
\left\|M\left(\epsilon_{M}\right)-M(0)\right\|_{\infty}=0.14
$$

or a relative difference of

$$
\left\|M\left(\epsilon_{M}\right)-M(0)\right\|_{\infty} /\|M(0)\|_{\infty}=2.28 \times 10^{-i} .
$$

This change in $M$ leads to the following relative changes in the computed eigendecomposition of $M(\delta)=Q(\delta) \Lambda(\delta) Q(\delta)^{-1}$ :

$$
\begin{aligned}
\left\|\Lambda\left(\epsilon_{M}\right)-\Lambda(0)\right\|_{\infty} /\|\Lambda(0)\|_{\infty} & =1.89 \times 10^{11} \\
\left\|Q\left(\epsilon_{M}\right)-Q(0)\right\|_{\infty} /\|Q(0)\|_{\infty} & =1.10 \\
\left\|Q\left(\epsilon_{M}\right)^{-1}-Q(0)^{-1}\right\|_{\infty} /\left\|Q(0)^{-1}\right\|_{\infty} & =0.32
\end{aligned}
$$

In the latter cases, the absolute errors are

$$
\begin{aligned}
\left\|Q\left(\epsilon_{M}\right)-Q(0)\right\|_{\infty} & =1.62 \times 10^{3} \\
\left\|Q\left(\epsilon_{M}\right)^{-1}-Q(0)^{-1}\right\|_{\infty} & =0.025
\end{aligned}
$$

The residual error for the computed right eigenpairs is

$$
\left\|M(0) Q\left(\epsilon_{M}\right)-Q\left(\epsilon_{M}\right) \Lambda\left(\epsilon_{M}\right)\right\|_{\infty} /\left\|\Lambda\left(\epsilon_{M}\right)\right\|_{\infty}=3.85 \times 10^{-4} .
$$

By any of these measurements, we see that a very small change in $H(\delta)$ leads to a significantly larger error in its computed eigendecomposition. An ill-conditioned problem in which deflation takes place could be even more inaccurate. As these errors are caused by ill-condition, there is no reason to expect a substantially different result when the QL method used for our experiments is replaced by root-finding.

If an inaccurate subproblem solution is detected, the matrix could be divided at a different point and the ill-conditioned submatrix replaced with a new submatrix. Despite the additionai work involved, this approach cannot guarantee new subproblems with better condition than the original ones. Furthermore, efficient divide and conquer methods typically recursively subdivide the original matrix more than once to form a 
tree of updating problems $[12,22,24]$. Even if the smanıst submatrices at the leaves of the tree are well-conditioned with respect to the eigenproblem, larger submatrices farther up the tree may not be. To correct for ill-conditioning at level $k$ of the tree, it would be necessary to redivide the matrix and repeat the updating procecure for all levels of the tree from the leaves to level $k$. Again, this redivision could not guarantee improved condition of the level $k$ problerns. To make matters worse, ill-conditioning could be introduced in the new problems below level $k$.

\section{Conclusion}

We have seen that the divide and conquer method that has been so successfully applied to other matrix problems can fall short when extended to the nonsymmetric eigenvalue problem. The speed and accuracy of the other methods rely largely on the availability of a fast and globally convergent root-finder and on the prevalence and ease of deflation. However, there appears to be no equivalent root-finder for the nonsymmetric case, and deflation of of the nonsymmetric problem may not be as advantageous as in the symmetric case. Furthermore, if it is necessary to compute the left eigenvectors from the right eigenvectors to maintain accuracy, the eigenvector computation may become inefficient, especially in parallel.

The greatest danger with the divide and conquer method, howernr, lies in its potential instability. Even if the original matrix is well-conditioned with respect to the eigenproblem, an ili-conditioned submatrix can be created at any level of updating. Thus, even small errors introduced by tearing, deflation, or updating can lead to large errors in the computed eigendecomposition. Because the original matrix is never used in the updating procedure, there is no opportunity to correct an error introduced by an ill-conditioned submatrix. Two divide and conquer methods that employ the matrix original matrix $T$ in the updating procedure and appear to overcome poor intermediate results are discussed in $[11,25]$.

\section{Acknowledgements}

The author thanks Loyce Adams, Peter Arbenz, Jack Dongarra, and Majed Sidani for several helpful discussions and thanks Bob Manchek for preparing Figures 1 and 2.

\section{References}

[1] L. Adams and P. Arbenz. Towards a divide and conquer algorithm for the real nonsymmetric eigenvalue problem. Department of Applied Mathematics, University of Washington, Research Report Preprint.

[2] G.S. Ammar, D. Cheng, W. Dayawansa, and C. Martin. Identification of linear systems by Prony's method. In Robust Control of Linear Systems and Nonlinear Control, pages 483-488, 1990.

[3] G.S. Ammar, W. Dayawansa, and C. Martin. Exponential interpolation: theory and numerical algorithms. To appear in J. Appl. Math. Comput. 
[4] G.S. Ammar, L. Reichel, and D.C. Sorensen. An implementation of a divide and conquer method for the unitary eigenproblem. To appear in ACM TOMS.

[5] J. Barlow. Error analysis of update methods for the symmetric eigenvalue problem. To appear in SIAM J. Matrix Anal. Appl.

[6] J.R. Bunch, C.P. Nielsen, and D.C. Sorensen. Rank-one modification of the symmetric eigenproblem. Numer. Math., 31:31-48, 1978.

[7] G.F. Carrier, M. Krook, and C.E. Pearson. Functions of a Complex Variable. McGraw-Hill Book Company, 1966.

[8] J.J.M. Cuppen. A divide and conquer method for the symmetric tridiagonal eigenproblem. Numer. Math., 36:177-95, 1981.

[9] J. Dongarra, G.A. Geist, and C.H. Romine. Computing the eigenvalues and eigenvectors of a general matrix by reduction to tridiagonal form. Technical Report ORNL/TM-11669, Oak Ridge National Laboratory, 1990.

[10] J. Dongarra, S. Hammarling, and D. Sorensen. Block reduction of matrices to condensed form for eigenvalue computations. Journal of Computational and Applied Mathematics, 27:215-227, 1989.

[11] J. Dongarra and M. Sidani. A divide and conquer method for the nonsymmtric eigenvalue problem. Department of Computer Science, University of Tennessee, Research report in preparation.

[12] J.J. Dongarra and D.C. Sorensen. A fully parallel algorithm for the symmetric eigenvalue problem. SIAM J. Sci. Stat. Comput., 8:s139-s154, 1987.

[13] K. Gates. University of Washington, PhD thesis in preparation, 1991.

[14] G.A. Geist. Reduction of a general matrix to tridiagonal form. Technical Report ORNL/TM-10991, Oak Ridge National Laboratory, 1989.

[15] G.A. Geist, A.Lu, and E.L. Wachspress. Stabilized reduction of an arbitrary matrix to tridiagonal form. Technical Report ORNL/TM-11089, Oak Ridge National Laboratory, 1989.

[16] G.A. Geist and G.J. Davis. Finding eigenvalues and eigenvectors of unsymmetric matrices using a distributed-memory multiprocessor. Technical Report ORNL/TM-10938, Oak Ridge National Laboratory, 1988.

[17] P.E. Gill, W. Murray, and M.H. Wright. Practical Optimization. Academic Press, 1981.

[18] G.H. Golub. Some modified matrix eigenvalue problems. SIAM Review, 15:318$34,1973$.

[19] G.H. Golub and C.F. Van Loan. Matrix Computations. The Johns Hopkins Press, Baltimore, MD, 2nd edition, 1989. 
[20] P. Henrici. Applied and Computational Complex Analysis. John Wiley and Sons, 1974.

[21] A.S. Householder. The Numerical Treatment of a Single Nonlinear Equation. McGraw-Hill, 1970.

[22] I.C.F. Ipsen and E.R. Jessup. Solving the symmetric tridiagonal eigetuvalue problem on the hypercube. SIAM J. Sci. Stat. Comput., Vol. 11, No. 2, pages 203-229, 1990.

[23] E.R. Jcssup and I.C.F. Ipsen. Improving the accuracy of inverse iteration. To appear in SIAM J. Sci. Stat. Comput.

[24] E.R. Jessup and D.C. Sorensen. A parallel algorithm for computing the singular value decomposition of a matrix. Technical Report ANL/MCS-TM-102, Argonne National Laboratory, 1987.

[25] T.Y. Li, Z. Zeng, and L. Cong. Solving eigenvalue problems of real nonsymmetric matrices with real homotopies. Dept. Mathematics and Dept. Computer Science, Michigan State University, Research Report Preprint, 1990.

[26] S. Lo, B. Phillipe, and A. Sameh. A multiprocessor algorithm for the symmetric tridiagonal eigenvalue problem. SIAM J. Sci. Stat. Comput., 8:s155-s165, 1987.

[27] C. Moler, J. Little, S. Bangert, and S. Kleiman. Pro-Matlab User's Guide. The MathWorks, Inc., 1987.

[28] D.P. O'Leary and G.W. Stewart. Computing the eigenvalues and eigenvectors of symmetric arrowhead matrices. J. Comp. Phys., 90:497-505, 1990.

[29] B.T. Smith, J.M. Boyle, J.J. Dongarra, B.S. Garbow, Y. Ikebe, V.C. Klema, and C.B. Moler. Matrix Eigensystem Routines-EISPACK Guide, Lecture Notes in Computer Science, Vol. 6, 2nd edition. Springer-Verlag, 1976.

[30] D.C. Sorensen and P. Tang. On the orthogonality of eigenvectors computed by divide and conquer techniques. To appear in SIAM J. Numerical Analysis.

[31] J.H. Wilkinson. The Algebraic Eigenvalue Problem. Clarendon Press, Oxford, 1965. 


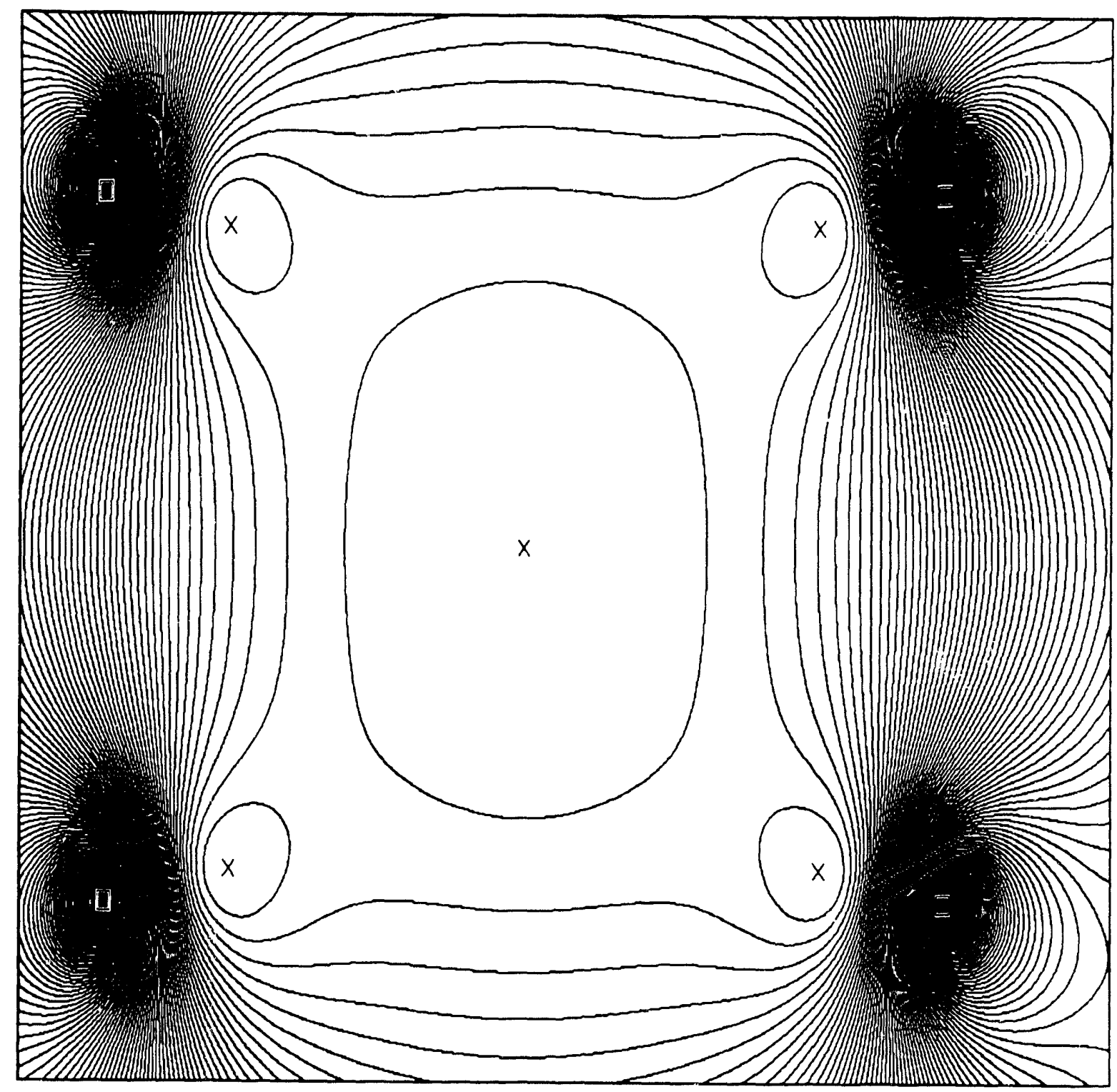

Figure 1: Contour plot of $\log (|g(\lambda)|)$ for the $5 \times 5$ matrix $T_{5}$. Poles are marked with boxes, and roots are marked with $\times$ 's 


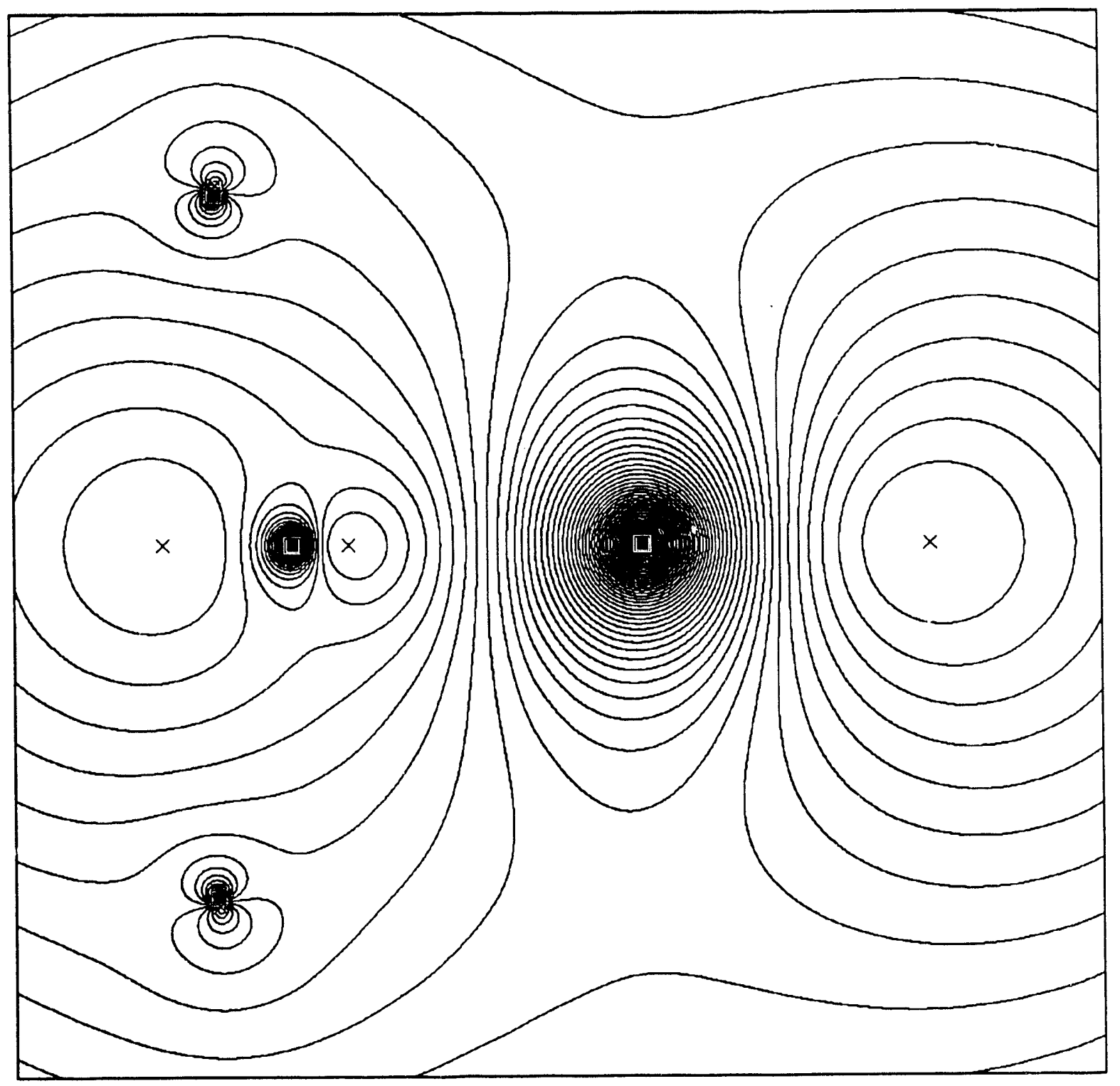

Figure 2: Contour plot of $\log (|g(\lambda)|)$ for a random $5 \times 5$ matrix. Poles are marked with boxes, and roots are marked with $\times$ 's 
ORNL/TM-11903

\section{INTERNAL DISTRIBUTION}

$\begin{aligned} 1 . & \text { B. R. Appleton } \\ 2-3 . & \text { T. S. Darland } \\ 4 . & \text { E. F. D' Azevedo } \\ 5 . & \text { J. M. Donato } \\ 6 . & \text { J. J. Dongarra } \\ 7 . & \text { G. A. Geist } \\ 8-12 . & \text { E. R. Jessup } \\ 13 . & \text { M. R. Leuze } \\ 14 . & \text { E. G. Ng } \\ 15 . & \text { C. E. Oliver } \\ 16 . & \text { B. W. Peyton } \\ 17-21 . & \text { S. A. Raby }\end{aligned}$

22. C. H. Romine

23. T. H. Rowan

24-28. R. F. Sincovec

29-33. R. C. Ward

34. P. H. Worley

35. Central Research Library

36. ORNL Patent Office

37. K-25 Applied Technology Library

38. Y-12 Technical Library

39. Laboratory Records - RC

40-41. Laboratory Records Department

\section{EXTERNAL DISTRIBUTION}

42. Loyce Adams, Department of Applied Mathematics, University of Washington, Seattle, WA 98195

43. Gregory Ammar, Northern Illinois University, Department of Mathematical Sciences, DeKalb, IL 60115

44. Peter Arbenz, ETH Zentrum Institut fur Wissenschaftlisches, Rechnen 8092, Zurich, Switzerland

45. Cleve Ashcraft, Boeing Computer Services, P.O. Box 24346, M/S 7L-21, Seattle, WA 98124-0346

46. Donald M. Austin, 6196 EECS Building, University of Minnesota, 220 Union Street, S.E., Minneapolis, MN 55455

47. Jesse L. Barlow, Department of Computer Science, Pennsylvania State University, University Park, PA 16802

48. Edward H. Barsis, Computer Science and Mathematics, P. O. Box 5800, Sandia National Laboratories, Albuquerque, NM 87185

49. Christopher Beattie, Department of Mathematics, Virginia Polytechnic Institute and State University, Blacksburg, VA 24061

50. Chris Bischof, Mathematics and Computer Science Division, Argonne National Laboratory, 9700 South Cass Ave., Argonne, IL 60439

51. Ake Bjorck, Department of Mathematics, Linkoping University, S-581 83 Linkoping, Sweden 
52. Jean R. S. Blair, Department of Computer Science, Ayres Hall, University of Tennessee, Knoxville, TN 37996-1301

53. Daniel Boley, Department of Computer Science, University of Minnesota, 200 Union Street, S.E., Rm 4-192, Minneapolis, MN 55455

54. Roger W. Brockett (EPMD Advisory Committee), Wang Professor of Elecirical Engineering and Computer Science, Division of Applied Sciences, Harvard University, Cambridge, MA 02138

55. Bill L. Buzbee, Scientific Computing Division, National Center for Atmospheric Research, P.O. Box 3000, Boulde", CO 80307

56. Ralph Byers, University of Kansas, Department of Mathematics, Snow Hall, Lawrence, KS 66045-2142

57. John Cavallini, Office of Scientific Computing, Office of Energy Research, ER-7, Germantown Building, U.S. Department of Energy, Washington, DC 20545

58. Tony Chan, Department of Mathematics, University of California, Los Angeles, 405 Hilgard Ave., Los Angeles, CA 90024

59. Eleanor Chu Department of Computer Science University of Waterloo Waterloo Ontario, Canada N2L 3G1

60. Melvyn Ciment, National Science Foundation, 1800 G Street, N.W., Washington, DC 20550

61. Thomas Coleman, Department of Computer Science, Comell University, Ithaca, NY 14853

62. John M. Conroy, Supercomputer Research Centcr, 17100 Science Drive, Bowie, MD $20715-4300$

63. Jane K. Cullum, IBM T. J. Watson Research Center, P.O. Box 218, Yorktown Heights, NY 10598

64. George Cybenko, Center for Supercomputing Research and Development, University of Illinois, 104 S. Wright Street, Urbana, IL 61801-2932

65. George J. Davis, Department of Mathematics, Georgia State University, Atlanta, GA 30303

66. James W. Demmel, Computer Science Division, University of California, Berkeley, CA 94720

67. John J. Dorning (EPMD Advisory Committee), Department of Nuclear Engineering Physics, Thomton Hall, McCormack Rd., University of Virginia, Charlottesville, VA 22901

68. Iain S Duff, Numerical Analysis Group, Central Computing Department, Atlas Centre, Rutherford Appleton Laboratory, Didcot, Oxon, OX11 0QX England 
69. Patricia Eberlein, Department of Computer Science, SUNY at Buffalo, Buffalo, NY 14260

70. Stanley Eisenstat, Departmeni of Computer Science, Yale University, P.O. Box 2158 Yale Station, New Haven, CT 06520

71. Lars Elden, Department of Mathematics, Linkoping University, 58183 Linkoping, Sweden

72. Howard C. Elman, Computer Science Department, University of Maryland, College Park, MD 20742

73. Albert M. Erisman, Boeing Computer Services, P.O. Box 24346, M/S 7L-21, Seattle, WA $98124-0346$

74. Geoffrey C. Fox, Booth Computing Center, 158-79 Califormia Institute of Technology, Pasadena, CA 91125

75. Paul O. Frederickson, NASA Ames Research Center, RIACS, M/S T045-1, Moffett Field, CA 94035

76. Robert E. Funderlic, Department of Computer Science, North Carolina State University, Raleigh, NC 27650

77. Dennis B. Gannon, Computer Science Department, Indiana University, Bloomington, IN 47405

78. David M. Gay, Bell Laboratories, 600 Mountain Ave., Murray Hill, NJ 07974

79. C. William Gear, Computer Science Department, University of Illinois, Urbana, IL 61801

80. W. Morven Gentleman, Division of Electrical Engineering, National Research Council, Building M-50, Room 344, Montreal Rd., Ottawa, Ontario, Canada K1 A 0R8

81. J. Alan George, Vice President Academic and Provost, Needles Hall, University of Waterloo, Waterloo, Ontaiio, Canada N2L 3G1

82. Gene H. Golub, Department of Computer Science, Stanford University, Stanford, CA 94305

83. Anne Greenbaum, Courant Institute, 251 Mercer Street, New York, NY 10012

84. John Gustafson, Ames Laboratory, Iowa Siate University, Ames, IA 50011

85. Sven Hammarling, Numerical Algorithms Group Ltd., Wilkinson House, Jordan Hill Road, Oxford OX2 8DR, United Kingdom

86. Per Christian Hansen, UNI*C Lyr .jy, Building 305, Technical University of Denmar's, DK-2800 Lyngby, Denmark

87. Richard Hanson, IMSL Inc., 2500 Park West Tower One, 2500 City West Boulevard, Houston, TX 77042-3020 
88. Michael T. Heath, National Center for Supercomputing Applications, 4157 Beckman Institute, University of Ilinois, 405 North Mathews Avenue, Urbana, IL 61801-2300

89. Don E. Heller, Physics and Computer Science Department, Shell Development Co., P.O. Box 481, Houston, TX 77001

90. Nicholas J. Higham, Department of Mathematics, University of Manchester, Grt Manchester, M13 9PL, England

91. Charles J. Holland, Air Force Office of Scientific Research, Building 410, Bolling Air Force Base, Washington, DC 20332

92. Ilse Ipsen, Department of Computer Science, Yale University, P.O. Box 2158 Yale Station, New Haven, CT 06520

93. Lennart Johnsson, Thinking Machines Inc., 245 First Street, Cambridge, MA 021421214

94. Harry Jordan, Department of Electrical and Computer Engineering, University of Colorado, Boulder. CO 80309

95. Bo Kagstrom, Institute of Information Processing, University of Umea, 5-901 87 Umea, Sweden

96. Malvin H. Kalos, Comell Theory Center, Engineering and Theory Center Building, Comell University, Ithaca, NY 14853-3901

97. Hans Kaper, Mathematics and Computer Science Division, Argonne National Laboratory, 9700 South Cass Ave., Argonne, IL 60439

98. Linda Kaufman, Bell Laboratories, 600 Mountain Avenue, Murray Hill, NJ 07974

99. Kenneth Kennedy, Department of Computer Science, Rice University, P.O. Box 1892, Houston, TX 77005

100. Thomas Kitchens, Department of Energy, Scientific Computing Staff, Office of Energy Research, ER-7, Office G-236 Germantown, Washington, DC 20585

101. Richard Lau, Code 1111MA, 800 N. Quincy Street, Boston Tower 1, Arlington, VA 22217-5000

102. Alan J. Laub, Department of Electrical and Computer Engineering, University of California, Santa Barbara, CA 93106

103. Robert L. Launer, Army Research Office, P.O. Box 12211, Research Triangle Park, NC 27709

104. Peter D. Lax, Courant Institute of Mathematical Sciences, New York University, 251 Mercer Street, New York, NY 10012

105. James E. Leiss (EPMD Advisory Committee), R:. 2 Box 142C, Broadway, VA 22815

106. John G. Lewis, Boeing Computer Services, P.O. Box 24346, M/S 7L-21, Seattle, WA 98124-0346 
107. Richard J. Littlefield, Pacific Northwest Laboratory, P.O. Box 999, Richland, WA 99532

108. T. Y. Li, Department of Mathematics, Michigan State University, E. Lansing, MI 48824-1027

109. Joseph Liu, Department of Computer Science, York University, 4799 Keele Street, North York, Ontario, Canada M3J 1P3

110. Franklin Luk, School of Electrical Engineering, Comell University, Ithaca, NY 14853

111. Thomas A. Manteuffel, Department of Mathematics, University of Colorado Denver, Denver, CO 80202

112. Volker L. Mehrmann, Universitaet Bielefeld, Fakulaet fuer Mathematik, Postfach 8640, D-4800, Bielefeld 1, Germany

113. Paul C. Messina, Mail Code 158-79, California Institute of Technology, 1201 E. Califomia Boulevard, Pasadena, CA 91125

114. James McGraw, Lawrence Livermore National Laboratory, L-306, P.O. Box 808 , Livermore, CA 94550

115. Cleve Moler, The Mathworks, 325 Linfield Place, Menlo Park, CA 94025

116. Neville Moray (EPMD Advisory Committee), Department of Mechanical and Industrial Engineering, University of Illinois, 1206 West Green Street, Urbana, IL 61801

117. Brent Morris, National Security Agency, Ft. George G. Meade, MD 20755

118. Dianne P. O'Leary, Computer Science Department, University of Maryland, College Park, MD 20742

119. Beth Ong, Department of Mathematics, University of California, Los Angeles, 405 Hilgard Ave., Los Angeles, CA 90024

120. James M. Ortega, Department of Applied Mathematics, Thornton Hall, University of Virginia, Charlottesville, VA 22903

121. Crris Paige, McGill University, School of Computer Science, McConnell Engineering Building, 3480 University Street, Montreal, Quebec, Canada H3A 2A7

122. Beresford N. Parlett, Department of Mathematics, University of Califomia, Berkeley, CA 94720

123. Merrell Patrick, Department of Computer Science, Duke University, Durham, NC 27706

124. Dan Pierce, B.jeing Computer Services, P.O. Box 24346, M/S 7L-21, Seattle, WA 98124-0346

125. Robert J. Plemmons, Departments of Mathematics and Computer Science, North Carolina State University, Raleigh, NC 27650

126. Jesse Poore, Department of Computer Science, Ayres Hall, University of Tennessee, Knoxville, TN 37996-1301 
127. Alex Pothen, Department of Computer Science, Pennsylvania State University, University Park, PA 16802

128. Yuanchang Qi, IBM European Petroleum Application Center, P.O. Box 585, N-4040 Hafrsfjord, Norway

129. Giuseppe Radicati di Brozolo, IBM European Center for Scientific and Engineering Computing, via Giorgione 159, I-00147 Roma, Italy

130. Lothar Reichel, Kent State University, Department of Mathematics and Computer Science, Kent, OH 44242

131. John K. Reid, Numerical Analysis Group, Central Computing Department, Atlas Centre, Rutherford Appleton Laboratory, Didcot, Oxon OX11 0QX, England

132. Werner C. Rheinboldt, Department of Mathematics and Statistics, University of Pittsburgh, Pittsburgh, PA 15260

133. John R. Rice, Computer Science Department, Purdue University, West Lafayette, IN 47907

134. Garry Rodrigue, Numerical Mathematics Group, Lawrence Livermore Laboratory, Livermore, CA 94550

135. Donald J. Rose, Department of Computer Science, Duke University, Durham, NC 27706

136. Axel Ruhe, Department of Computer Science, Chalmers University of Technology, S-41296 Goteborg, Sweden

137. Joel Saltz, ICASE, MS 132C, NASA Langley Research Center, Hampton, VA 23665

138. Ahmed H. Sameh, Center for Supercomputing R\&D, 1384 W. Springfield Avenue, University of Illinois, Urbana, IL 61801

139. Michael Saunders, Systcms Optimization Laboratory, Operations Research Department, Stanford University, Stanford, CA 94305

140. Robert Schreiber, RIACS, Mail Stop 230-5, NASA Ames Research Center, Moffet Field, CA 94035

141. Martin H. Schultz, Department of Computer Science, Yale University, P.O. Box 2158 Yale Station, New Haven, CT 06520

142. Majed Sidani, Department of Computer Science, Ayres Hall, University of Tennessee, Knoxville, TN 37996-1301

143. David S. Scott, Intel Scientific Computers, 15201 N.W. Greenbrier Pkwy., Beaverton, OR 97006

144. Kermit Sigmon, Department of Mathematics, University of Florida, Gainesville, FL 32611

145. Horst Simon, Mail Stop 258-5, NASA Ames Research Center, Moffett Field, CA 94035 
146. Anthony Skjellum, Lawrence Livermore National Laboratory, 7000 East Ave., L-316, P.O. Box 808, Livermore, CA 94551

147. Danny C. Sorensen, Department of Mathematical Sciences, Rice University, P. O. Box 1892, Houston, TX 77251

148. Rick Stevens, Mathematics and Computer Science Division, Argonne National Laboratory, 9700 South Cass Ave., Argonne, IL 60439

149. G. W. Stewart, Computer Science Department, University of Maryland, College Park, MD 20742

150. Paul N. Swartztrauber, National Center for Atmospheric Research, P.O. Box 3000 , Boulder, CO 80307

151. Daniel B. Szyld, Department of Computer Science, Duke University, Durham, NC 27706-2591

152. Philippe Toint, Department of Mathematics, University of Namur, NUNOP, 61 rue de Bruxelles, B-Namur, Belgium

153. Bernard Tourancheau, LIP, ENS-Lyon, 69364 Lyon cedex 07, France

154. Lloyd N. Trefethen, Massachusetts Institute of Technology, Room 2-383, Cambridge, MA 02139

155. Anna Tsao, Supercomputing Research Center, 17100 Science Drive, Bowie, MD 20715-4300

156. Hank Van der Vorst, Department of Techn. Mathematics and Computer Science, Delft University of Technology, P.O. Box 356, NL-2600 AJ Delft, The Netherlands

157. Charles Van Loan, Department of Computer Science, Cornell University, Ithaca, NY 14853

158. James M. Varah, Centre for Integrated Computer Systems Research, University of British Columbia, Office 2053-2324 Main Mall, Vancouver, British Columbia V6T 1W5, Canada

159. Robert G. Voigt, ICASE, MS 132-C, NASA Langley Research Center, Hampton, VA 23665

160. Phuong, Vu, Cray Research Inc., 1408 Northland Dr., Mendota Heights, MN 55120

161. Daniel D. Warner, Department of Mathematical Sciences, 0-104 Martin Hall, Clemson University, Clemson, SC 29631

162. D. S. Watkins, Department of Pure and Applied Mathematics, Washington State University, Pullman, WA 99164-2930

163. Mary F. Wheeler (EPMD Advisory Committee), Rice University, Department of Mathematical Sciences, P.O. Box 1892, Houston, TX 77251

164. Andrew B. White, Computing Division, Los Alamos National Laboratory, Los Alamos, NM 87545 
165. Margaret Wright, Bell Laboratories, 600 Mountain Ave., Murray Hill, NJ 07974

166. David Young, University of Texas, Center for Numerical Analysis, RLM 13.150, Austin, TX 78731

167. Hong Zhang, Department of Mathematical Sciences, Clemson University, Clemson, SC 29634-1907

168. Office of Assistant Manager for Energy Research and Development, U.S. Department of Energy, Oak Ridge Operations Office, P.O. Box 2001, Oak Ridge, TN 37831-8600

169-178. Office of Scientific \& Technical Information, P.O. Box 62, Oak Ridge, TN 37831 

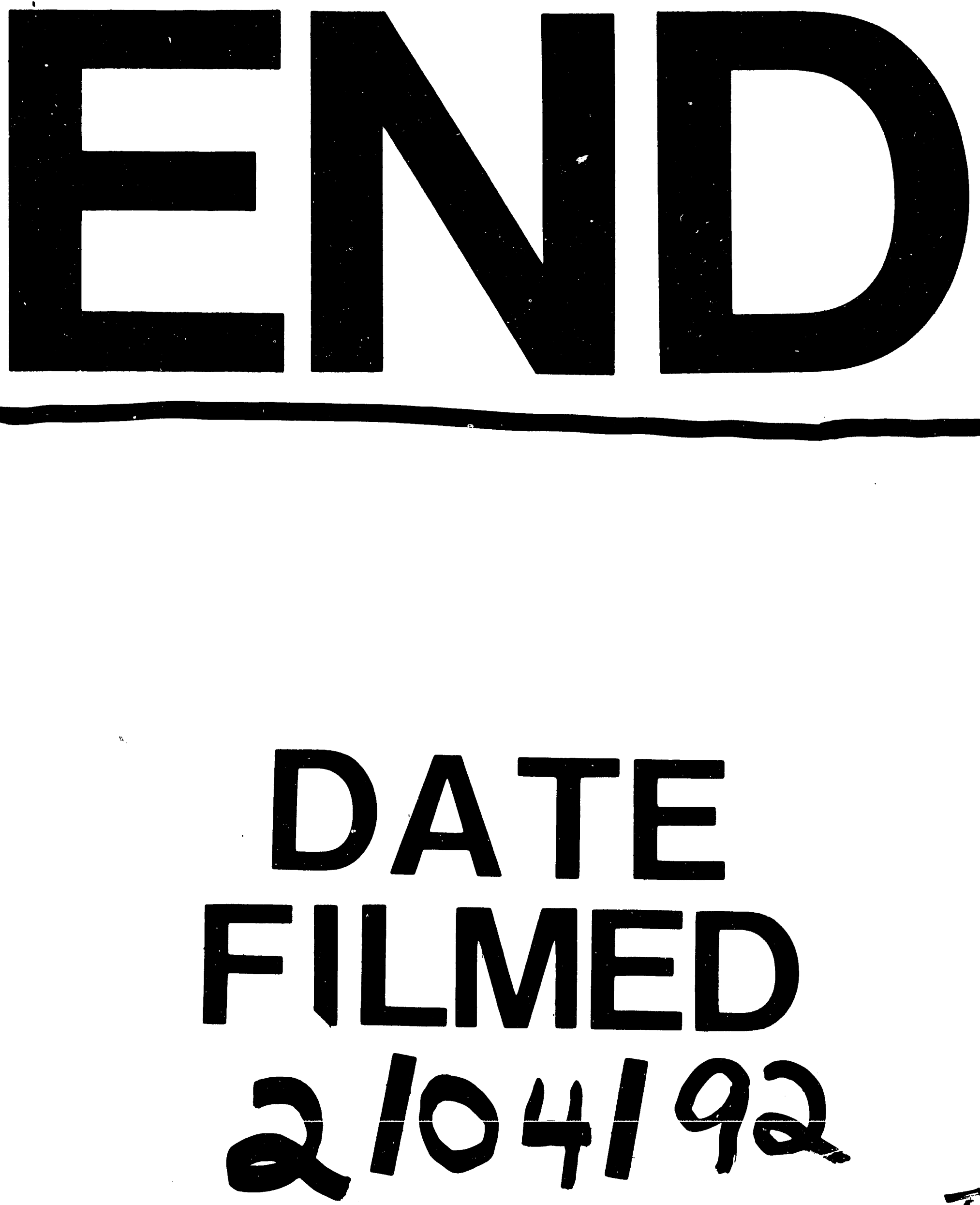

$I$ 
